\title{
Defects and gas sensing properties of carbon nanotube-based devices
}

\author{
S. Baldo ${ }^{1,2}$, V. Scuderi ${ }^{1}$, L. Tripodi ${ }^{1,2}$, A. La Magna ${ }^{1}$, S.G. Leonardi ${ }^{3}$, N. Donato ${ }^{3}$, G. Neri ${ }^{3}$, S. Filice ${ }^{4}$, \\ and S. Scalese ${ }^{1}$ \\ ${ }^{1}$ Istituto per la Microelettronica e Microsistemi, CNR, VIII Strada 5, 95121, Catania, Italy \\ ${ }^{2}$ Dipartimento di Fisica e Astronomia, Università degli Studi di Catania, Via S. Sofia, 95125, Catania, Italy \\ ${ }^{3}$ Dipartimento di Ingegneria Elettronica, Chimica e Ingegneria Industriale, Università degli Studi di Messina, \\ Contrada di Dio, Salita Sperone 31, Messina, Italy \\ ${ }^{4}$ Dipartimento di Chimica e Tecnologie Chimiche, Università della Calabria, Via P. Bucci, cubo 14/D, 87036, \\ Arcavacata di Rende (CS), Italy
}

Correspondence to: S. Baldo (salvatore.baldo@imm.cnr.it) and S. Scalese (silvia.scalese@imm.cnr.it)

Received: 30 July 2014 - Revised: 23 December 2014 - Accepted: 6 January 2015 - Published: 4 February 2015

\begin{abstract}
In this work we report on the development of back-gated carbon nanotube-field effect transistors (CNT-FETs), with CNT layers playing the role of the channel, and on their electrical characterisation for sensing applications. The CNTs have been deposited by electrophoresis on an interdigitated electrode region created on a $\mathrm{SiO}_{2} / \mathrm{Si}$ substrate. Different kinds of CNTs have been used (MWCNTs by arc discharge in liquid nitrogen and MWCNTs by chemical vapour deposition, CVD) and the electrical characterisation of the devices was performed in a $\mathrm{NH}_{3}$ - and $\mathrm{NO}_{2}$-controlled environment. Preliminary data have shown an increase in the channel resistance under $\mathrm{NH}_{3}$ exposure, whereas a decrease is observed after exposure to $\mathrm{NO}_{2}$, and the sensitivity to each gas depends on the kind of CNTs used for the device.

Furthermore, the defect formation by Si ion implantation on CNTs was investigated by high-resolution transmission electron microscopy (TEM) and Raman analysis. The behaviour observed for the different devices can be explained in terms of the interaction between structural or chemical defects in CNTs and the gas molecules.
\end{abstract}

\section{Introduction}

Since their discovery, carbon nanotubes (CNTs) have aroused great interest due to their exceptional properties related to their one-dimensional character (Deretzis et al., 2006) like a high current-carrying capacity, high thermal conductivity and reduced charge carrier scattering. The use of both individual nanotubes and CNT networks has been explored, depending on the specific applications (Zhou et al., 2002). Currently they are used in various technological applications, such as microelectronics or nanoelectronics, the automotive field, telecommunications, the aerospace industry, and the biomedical field.

Due to a very high surface-to-volume ratio, high electron mobility, great surface reactivity and high capability of gas adsorption, CNTs can be used as sensitive layers in gas sensors for environmental monitoring, industrial process con- trol and non-invasive biomedical analysis, by exploiting the changes in their electrical characteristics induced by surface chemical modifications (Zhang et al., 2008).

Defects in CNTs play a crucial role in the electronic, optical and mechanical properties (Scuderi et al., 2014), and they can be wanted or unwanted, depending on the kind of application: for example, for sensor applications, defected nanotubes are considered more desirable than ideal CNTs (Robinson et al., 2006; Neophytou et al., 2007), due to a larger interaction of the adsorbing species with defective sites. Of course, in order to take advantage of the defects presence, a suitable control on their generation, kind and amount has to be achieved. Furthermore, depending on the kind of defects, the binding energy should be evaluated and recovery mechanisms after gas molecules have been adsorbed on the CNT wall defects have to be investigated. 
In this work, back-gated carbon nanotube-field effect transistors (CNT-FETs) have been produced where a CNT network forms the channel of the FET, and the role of defects in the sensing properties, already present or induced by ion implantation on the CNT walls, has been investigated.

\section{Experimental}

Two types of CNTs were used to investigate the sensing properties of the devices produced: commercial MWCNTs synthesised by the Sigma Aldrich CoMoCAT ${ }^{\circledR}$ catalytic CVD process, hereafter called MWCNTs-CVD, and MWCNTs synthesised by arc discharge in liquid nitrogen $\left(\mathrm{LN}_{2}\right)$ and oxidised in $\mathrm{H}_{2} \mathrm{O}_{2}$ (diluted at $30 \%$ ) for $2 \mathrm{~h}$ in an ultrasonic bath (Bagiante et al., 2010; Scalese et al., 2010), hereafter called MWCNTs-LN 2 . The MWCNTs were deposited by the electrophoresis technique on an interdigitated electrode region created on $\mathrm{SiO}_{2} / \mathrm{Si} \mathrm{n}^{++}$type substrate (Baldo et al., 2014; Scuderi et al., 2012a), as reported in the scanning electron microscopy (SEM) image in Fig. 1a and b. In Fig. 1c and $\mathrm{d}$, transmission electron microscopy (TEM) images are reported for both kinds of nanotubes, showing relevant differences in the structural order. The conductive Si substrate is used as a back-gate contact. The FET structure is useful in order to investigate any possible changes in the CNT electrical properties induced, for example, by ion implantation processes.

A complete electrical characterisation has been carried out, both in air and in controlled gaseous environments $\left(\mathrm{NO}_{2}\right.$ and $\mathrm{NH}_{3}$ ), using two source meters (SMU) in a common source configuration, the Keithley 6487 for gate biasing and the Keithley 2400 for source and drain biasing (Fig. 2a). Both instruments, connected to a computer by the general purpose interface bus (GPIB), are totally controlled by MATLAB software. In this way a complete parameter analyser is obtained. The devices have been connected to the instruments with an appropriate test fixture (Fig. 2b) that gives the possibility of performing measurements in air and gaseous environments through an appropriate chamber connected to the gas system (Fig. 2c).

Understanding of the role of defects in the gas sensing properties of CNTs is of great relevance for the improvement of the sensitivity of CNT-based gas sensors. Defect formation by $\mathrm{Si}$ ion implantation on MWCNTs- $\mathrm{LN}_{2}$ was also investigated. The samples were implanted with $\mathrm{Si}^{+}$ions at $180 \mathrm{keV}$. The used energy was fixed so that the silicon implanted profile was fully contained in the $\mathrm{SiO}_{2} / \mathrm{Si}$ substrate. The ion doses were $1 \times 10^{13}, 5 \times 10^{13}$ and $1 \times 10^{14} \mathrm{~cm}^{-2}$.

The number of carboxyl groups has been determined by thermo-gravimetric analysis (TGA) using a Perkin Elmer Pyris 6 TGA thermo-gravimetric analyser. After reaching a thermal equilibrium at $30^{\circ} \mathrm{C}$ for $5 \mathrm{~min}$, the samples are heated up to $900^{\circ} \mathrm{C}$ with a heating rate of $10^{\circ} \mathrm{min}^{-1}$. The structural characterisation of the samples was performed by
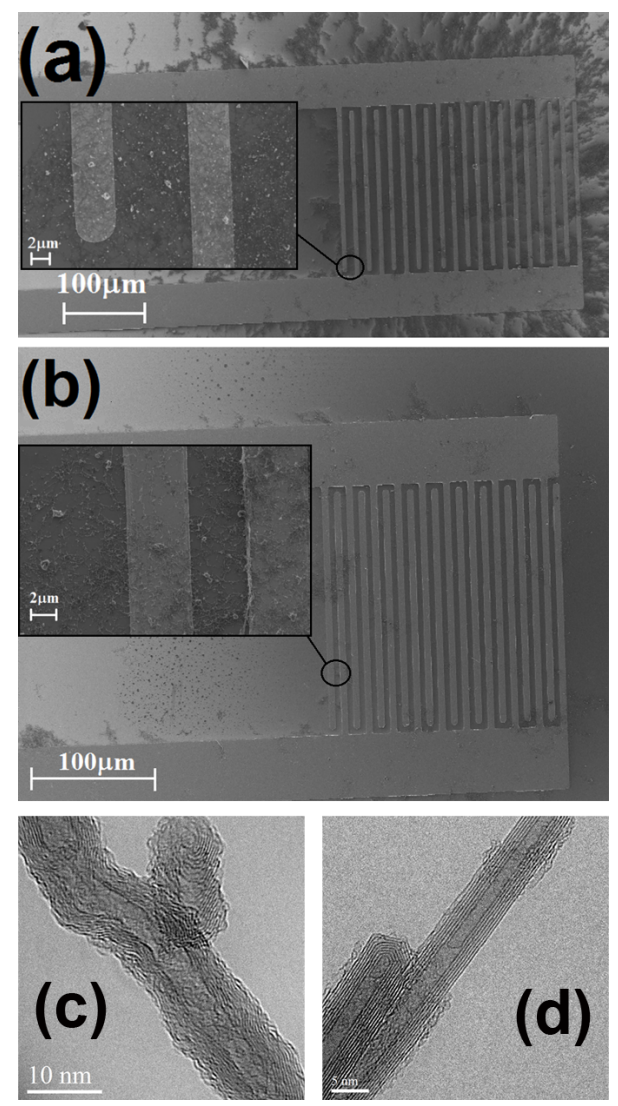

Figure 1. SEM images of devices with MWCNTs-CVD (a) and MWCNTs-LN 2 (b). In (c) and (d), TEM images of CNTs produced, respectively, by CVD and arc discharge are reported; the latter were treated by $\mathrm{H}_{2} \mathrm{O}_{2}$.

SEM, using a ZEISS SUPRA 35 FE-SEM system with a field emission electron gun, and by TEM, using a JEM 2010F JEOL microscope operating with an acceleration voltage of $200 \mathrm{kV}$.

Raman scattering has been excited by a $514.5 \mathrm{~nm}$ radiation coming from an Ar ion laser and the scattered light has been analysed by a single $460 \mathrm{~mm}$ monochromator (Jobin-Yvon HR460). Laser power was always kept below $10 \mathrm{~mW}$ at the sample to avoid its degradation, and the accumulation time was in the range of a few minutes.

\section{Results and discussion}

In Fig. 3 we report on the sensitivity of two kinds of devices fabricated, using respectively MWCNTs-LN 2 (black line) or commercial MWCNTs-CVD (red line) as the current channel, when they are exposed to $\mathrm{NH}_{3}$ (Fig. 3a) and $\mathrm{NO}_{2}$ (Fig. 3b). The sensitivity is expressed in terms of $\Delta R / R_{0}$, where $R_{0}$ is the channel resistance in dry air (reference resistance) and $\Delta R=R-R_{0}$, where $R$ is the channel resistance in $\mathrm{NH}_{3}$ or $\mathrm{NO}_{2}$, keeping the same bias conditions $\left(V_{\mathrm{gs}}=V_{\mathrm{ds}}=5 \mathrm{~V}\right)$. Electrical characterisation shows that the 

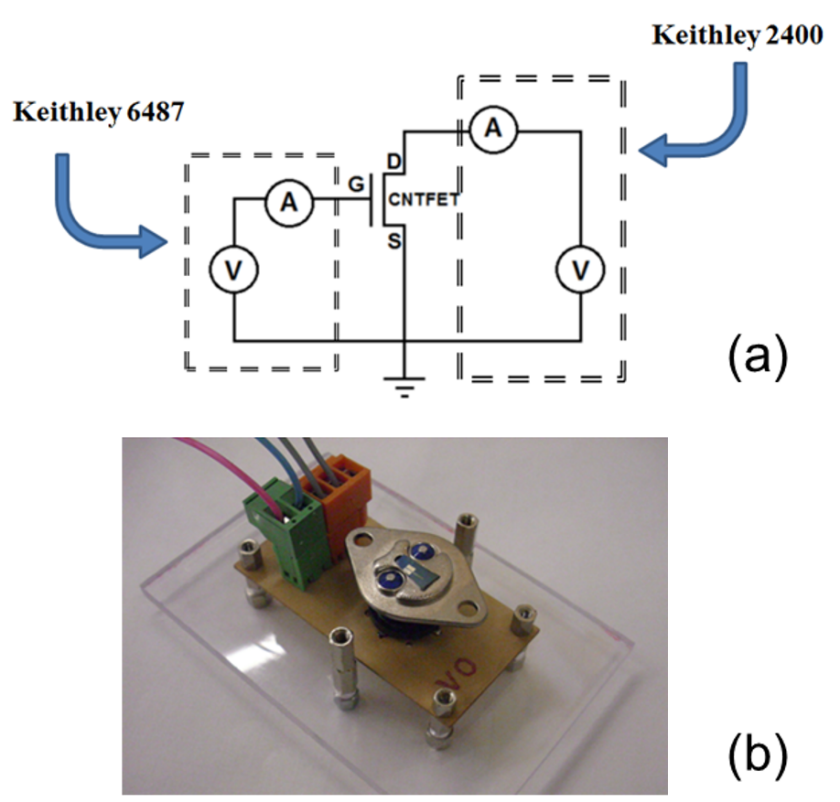

(b)

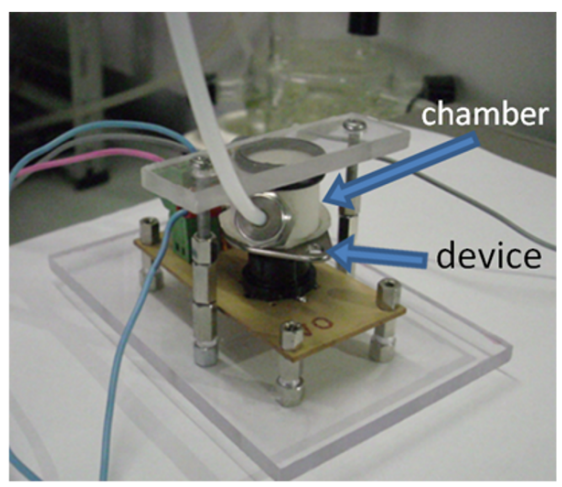

(c)

Figure 2. (a) Measurement set-up scheme; (b) test fixture; (c) test fixture and chamber for gas exposure.

exposure to $\mathrm{NH}_{3}$ induces an increase in the channel resistance as the concentration of ammonia is increased, and vice versa, during the exposure to $\mathrm{NO}_{2}$, a decrease in the channel resistance is noted as the $\mathrm{NO}_{2}$ concentration is increased. The response is attributed to the electrical charge transfer induced on the CNTs by the two kinds of molecules, which are electron donors $\left(\mathrm{NH}_{3}\right)$ or acceptors $\left(\mathrm{NO}_{2}\right)$ (Donato et al., 2011). The device with MWCNTs- $\mathrm{LN}_{2}$ shows a better sensitivity to $\mathrm{NH}_{3}$ compared to the device with MWCNTs-CVD (Fig. 3a), while the device with MWCNTs-CVD shows a better sensitivity to $\mathrm{NO}_{2}$ compared to the other one (Fig. 3b).

These results depend on the different interaction of the gaseous species with CNTs. In fact, the presence of the $\mathrm{COOH}$ groups on the CNT walls, in the case of MWCNTs$\mathrm{LN}_{2}$, favours an acid-base chemical interaction between the oxidised $\mathrm{CNT}$ and $\mathrm{NH}_{3}$ molecules. $\mathrm{NO}_{2}$ molecules instead are generally adsorbed by structural defects like vacancies, which are more abundant in MWCNTs-CVD. TGA results are shown in Fig. 4, expressed as weight $\%$ or derivative
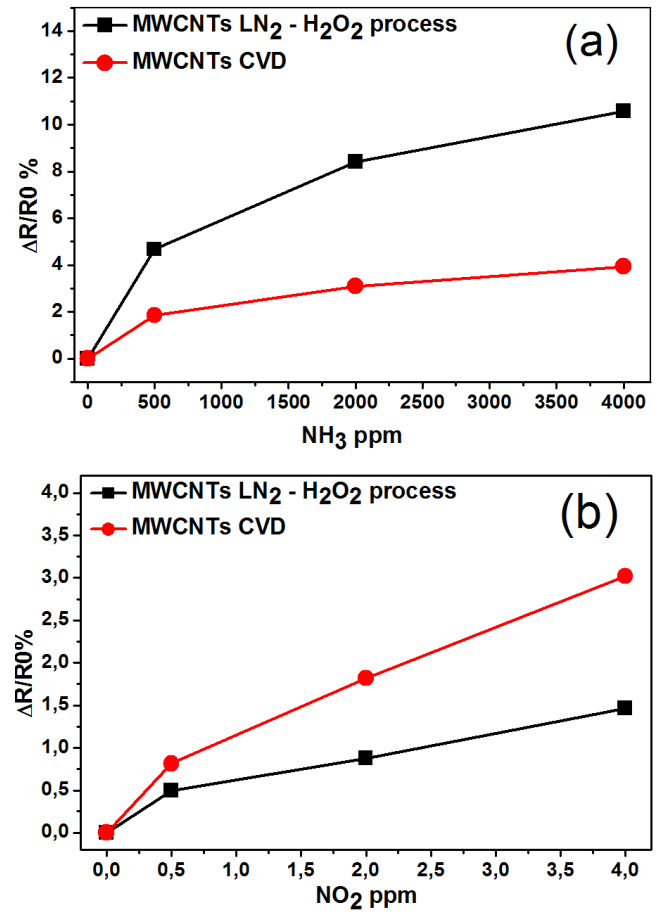

Figure 3. Sensitivity values $\left(\Delta R / R_{0}\right)$ for MWCNT-CVD and MWCNT-LN 2 devices to $\mathrm{NH}_{3}$ (a) and $\mathrm{NO}_{2}$ (b).
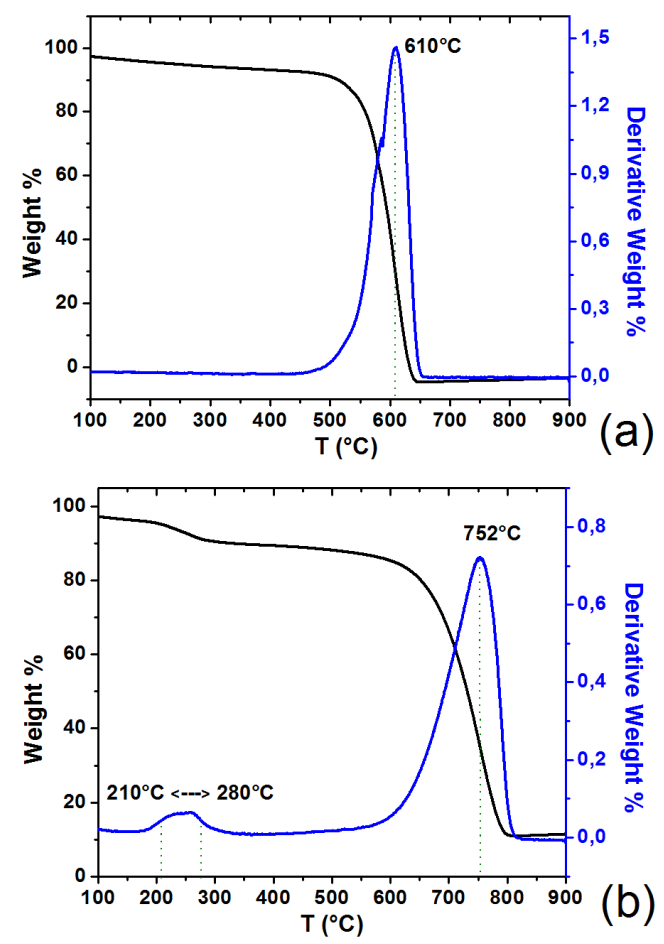

Figure 4. Thermo-gravimetric analysis of MWCNTs-CVD (a) and MWCNTs- $\mathrm{LN}_{2}$ (b). The values are expressed as weight $\%$ on the left axis or as derivative weight on the right axis. 

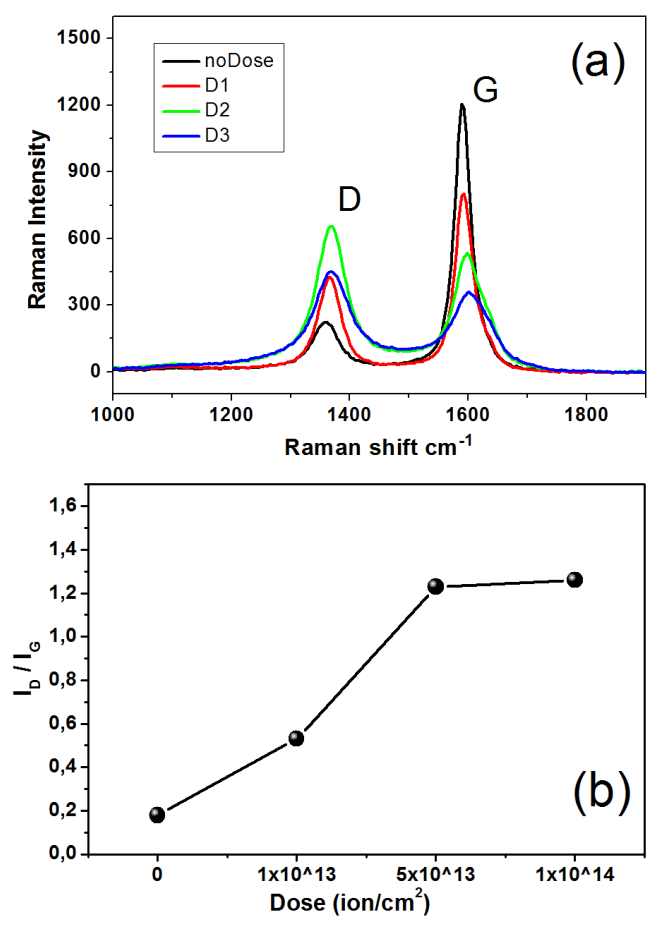

Figure 5. Raman spectra of the sample (a) not implanted (black line) and implanted with $\mathrm{Si}$ ion doses of $1 \times 10^{13} \mathrm{~cm}^{-2}$ (red line), $5 \times 10^{13} \mathrm{~cm}^{-2}$ (green line) and $1 \times 10^{14} \mathrm{~cm}^{-2}$ (blue line); (b) $I_{\mathrm{D}} / I_{\mathrm{G}}$ ratio vs. implant dose.

weight (left and right axes) as a function of temperature. A weight loss of $4 \%$ between 210 and $280^{\circ} \mathrm{C}$ (Fig. 4b) is observed in the case of MWCNTs- $\mathrm{LN}_{2}$, confirming the presence of carboxyl groups (Datsyuk et al., 2008). Conversely, in MWCNTs-CVD, no weight loss is observed in that range of temperatures (Fig. 4a). In both materials, no hydroxyl functionalities, usually degrading in the temperature range $350-500{ }^{\circ} \mathrm{C}$, are observed. Furthermore, the TGA shows that MWCNTs- $\mathrm{LN}_{2}$ are inherently less defective and therefore more stable than the other ones, since the total weight loss of carbon is obtained for higher temperatures with respect to the other kinds of nanotubes $\left(752\right.$ vs. $\left.610^{\circ} \mathrm{C}\right)$.

In order to investigate the role of defects in the CNT sensing properties, we have introduced structural defects by ion implantation on the CNTs that show a better structural quality (arc discharge synthesis). In particular, Si ion implantation has been performed at $180 \mathrm{keV}$ at room temperature with three doses: $1 \times 10^{13} \mathrm{~cm}^{-2}$ (called D1), $5 \times 10^{13} \mathrm{~cm}^{-2}$ (called D2) and $1 \times 10^{14} \mathrm{~cm}^{-2}$ (called D3). The reference sample, not exposed to ion implantation, is called D0.

After the ion implantation, the samples were analysed by Raman spectroscopy, in order to evaluate the structural order of the nanotubes. The spectra were acquired at different points of each sample in order to verify the uniformity of the material deposited on the area between the electrodes. The obtained spectra showed a very similar $I_{\mathrm{D}} / I_{\mathrm{G}}$ ratio for each
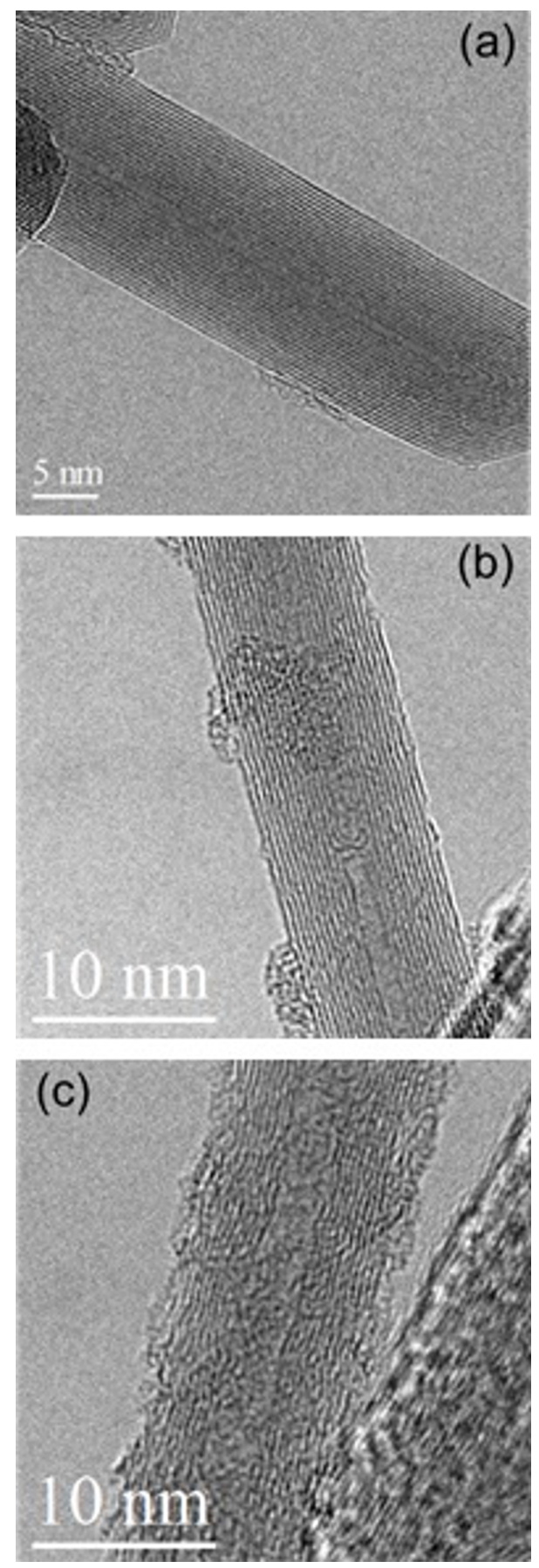

Figure 6. Typical CNT structures and defects observed by TEM for the samples (a) not implanted, (b) implanted with a $\mathrm{Si}$ ion dose of $1 \times 10^{13} \mathrm{~cm}^{-2}$, and (c) implanted with a Si ion dose of $1 \times 10^{14} \mathrm{~cm}^{-2}$.

sample, indicating good uniformity in the entire electrode area. In Fig. 5a we report just one of the acquired spectra for each sample. The spectra show the typical D band at around $1361 \mathrm{~cm}^{-1}$, due to defects present in the nanotubes, and the $\mathrm{G}$ band at around $1591 \mathrm{~cm}^{-1}$, related to the graphitic order of 


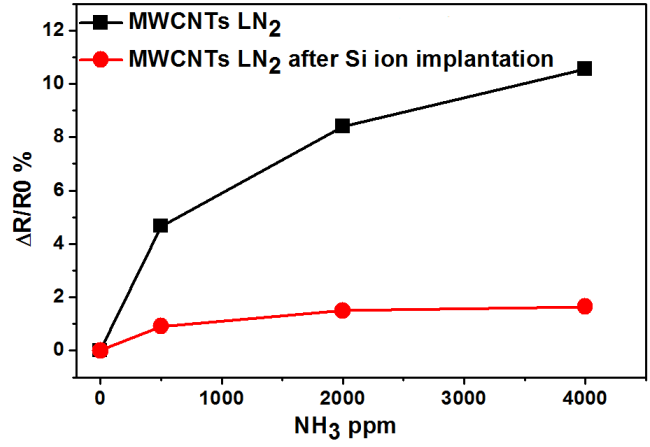

Figure 7. Comparison between $\Delta R / R_{0}$ values obtained for MWCNTs-LN2 and MWCNTs-CVD in $\mathrm{NH}_{3}$ before (black line) and after (red line) Si implantation $\left(V_{\mathrm{gs}}=V_{\mathrm{ds}}=5 \mathrm{~V}\right)$.

the nanotubes. Starting from the reference spectrum (black curve) related to sample D0, as the ion dose is increased, it is possible to observe an increase in the D peak intensity, a decrease in the $G$ peak intensity and an enlargement of both. The ratio between the $\mathrm{D}$ and $\mathrm{G}$ band intensities $\left(I_{\mathrm{D}} / I_{\mathrm{G}}\right)$ gives information on the graphitic order of $\mathrm{C}$ structures: in particular, a lower $I_{\mathrm{D}} / I_{\mathrm{G}}$ ratio means a better structural order. The $I_{\mathrm{D}} / I_{\mathrm{G}}$ ratio calculated from Fig. 5a is reported as a function of the implant dose in Fig. 5b. As expected, the CNT structural quality worsens for higher implant doses: the $I_{\mathrm{D}} / I_{\mathrm{G}}$ value increases from 0.6 for the sample not implanted up to 1.5 for the sample implanted with a dose of $1 \times 10^{14} \mathrm{~cm}^{-2}$.

The samples D0, D1 and D3 were also investigated by transmission electron microscopy in order to compare the damage degrees. The TEM images, reported in Fig. 6, show the effects of ion implantation, like vacancy formation and coalescence, shell burning, and diffusion of carbon atoms inside the structure. In particular, the damage is more evident for the sample implanted with the highest dose $(1 \times$ $10^{14} \mathrm{~cm}^{-2}$ ), shown in Fig. $6 \mathrm{c}$, in agreement with the results obtained by Raman spectroscopy.

The silicon ions that arrive on carbon nanotubes with an energy of $180 \mathrm{keV}$ can break the outer shell and induce migrations of carbon atoms on the structures. For the highest implant dose, the amorphous carbon arranges itself on the outer shell like a carbon overcoat (Fig. 6c).

The removal of carbon atoms from CNT walls leads to a rearrangement of the network from a hexagonal structure to a coherent structure also containing non-six-membered rings. In particular, the presence of pentagons and/or heptagons in the structure changes the curvature of graphene cylinders (Iijima et al., 1992; Scuderi et al., 2012b) and, furthermore, it is possible to see that external diameter shrink due to the continuous loss of atoms (Banhart et al, 2005).

In order to investigate the effect of defects induced by ion implantation (dose of $1 \times 10^{13} \mathrm{~cm}^{-2}$ ) on the sensing properties, an electrical characterisation of the CNT network before and after ion implantation was done in an $\mathrm{NH}_{3}$ gaseous envi-

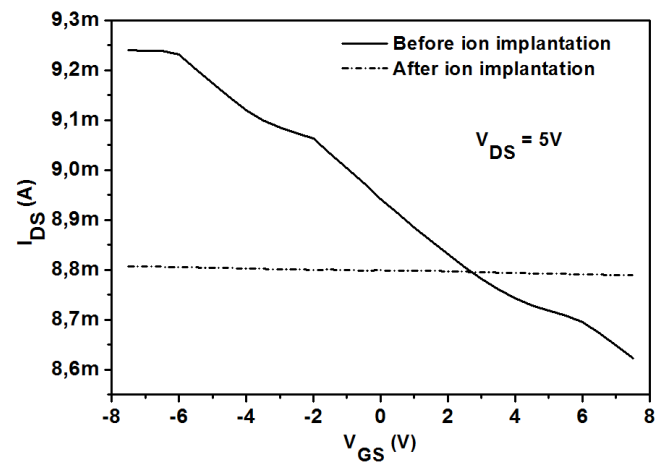

Figure 8. Dependence of $I_{\mathrm{ds}}$ on $V_{\mathrm{gs}}$ for the CNT-FET device before and after implantation (Si ion dose: $1 \times 10^{13} \mathrm{~cm}^{-2}$ ), for a fixed $V_{\mathrm{ds}}$ value of $5 \mathrm{~V}$.

ronment. In Fig. 7, we compare the sensitivity of the device, before (black line) and after (red line) ion implantation, keeping the same biasing conditions $\left(V_{\mathrm{gs}}=V_{\mathrm{ds}}=5 \mathrm{~V}\right)$ in terms of $\Delta R / R_{0}$. A worse response of the device is evidenced (red line) after ion implantation. Therefore, it looks like Si implantation has led to a deterioration in the CNT sensitivity to $\mathrm{NH}_{3}$ molecules.

We guess that the removal of carbon atoms from CNT walls and the consequent rearrangement of the hexagonal lattice, due to ion implantation, in some way also involves the - $\mathrm{COOH}$ groups responsible for the $\mathrm{NH}_{3}$ adsorption. In particular, the removal of or the change in the $-\mathrm{COOH}$ groups causes a reduction in the molecules' adsorption on the CNT walls and, therefore, a reduced sensitivity to $\mathrm{NH}_{3}$.

In Fig. 8 we report a comparison between the $I_{\mathrm{ds}}-V_{\mathrm{gs}}$ trans-characteristics obtained for the same device before and after ion implantation ( $\mathrm{Si}$ ion dose: $1 \times 10^{13} \mathrm{~cm}^{-2}$ ): the initial dependence of $I_{\mathrm{ds}}$ on $V_{\mathrm{gs}}$ disappears after implantation, thus indicating a transition of the electrical behaviour of CNTs from semiconducting to quasi-metallic. This behaviour is compatible with the removal of - $\mathrm{COOH}$ functionalities. Further experiments are still needed to understand these findings deeply.

\section{Conclusions}

In this work, back-gated CNT-based sensors have been developed and characterised using a CNT network as the FET channel. In particular, we have investigated the role of defects, already present or induced by ion implantation on the CNT walls, in the sensing properties. Electrical characterisation has shown that the exposure to $\mathrm{NH}_{3}$ induces an increase in the channel resistance as the concentration of ammonia is increased. The device with MWCNTs- $\mathrm{LN}_{2}$ shows a better sensitivity to $\mathrm{NH}_{3}$ compared to the device with MWCNTs-CVD. TGA shows the presence of carboxyl groups in the MWCNTs- $\mathrm{LN}_{2}$, confirming that the interaction between $\mathrm{CNTs}$ and $\mathrm{NH}_{3}$ occurs by $-\mathrm{COOH}$ groups present 
after treatment in $\mathrm{H}_{2} \mathrm{O}_{2}$. Vice versa, during the exposure to $\mathrm{NO}_{2}$, a decrease in the channel resistance was noted as the $\mathrm{NO}_{2}$ concentration is increased. In this case, the device with MWCNTs-CVD shows a better sensitivity to $\mathrm{NO}_{2}$ compared to the device with MWCNTs-LN 2 , showing that structural defects due to the CVD growth improve the $\mathrm{NO}_{2}$ sensing properties.

Furthermore, defect formation by $\mathrm{Si}$ ion implantation on MWCNTs- $\mathrm{LN}_{2}$ has been investigated. Raman analysis shows an increase of the $I_{\mathrm{D}} / I_{\mathrm{G}}$ ratio related to an increase in structural disorder, as expected. TEM analyses confirm these observations, showing the presence of large holes (vacancy agglomerations) on the CNT outer walls and the presence of amorphous carbon layers on the outer surface of the CNTs.

Electrical characterisation indicates that the Si ion implantation has reduced the CNT sensitivity to $\mathrm{NH}_{3}$. We guess that ion implantation, in some way, removes or alters the $-\mathrm{COOH}$ groups responsible for the $\mathrm{NH}_{3}$ adsorption.

Further studies have to be carried out in order to achieve the complete understanding of the observed behaviours and to tailor the structural properties of CNTs for the improvement of sensing properties of such CNT-based devices.

Acknowledgements. The authors acknowledge expert technical support by A. Marino for ion implantation processes, C. Bongiorno for TEM analysis, S. Di Franco for lithographic processes, N. Godbert for TGA analysis and the group of Prof. G. Compagnini for Raman spectroscopy.

This work has been funded by MIUR by means of the PON R\&C 2007-2013 national programme, project "Hyppocrates Sviluppo di Micro e Nano-Tecnologie e Sistemi Avanzati per la Salute dell'uomo" (PON02 00355).

Edited by: A. Romano-Rodriguez

Reviewed by: two anonymous referees

\section{References}

Bagiante, S., Scalese, S., Scuderi, V., D’Urso, L., Messina, E., Compagnini, G., and Privitera, V.: Role of the growth parameters on the structural order of MWCNTs produced by arc discharge in liquid nitrogen, Phys. Stat. Sol. B, 247, 884-887, 2010.

Baldo S., Scalese, S., Scuderi, V., Tripodi, L., La Magna, A., Romano, L., Leonardi, S. G., and Donato, N.: Correlation between structural and sensing properties of carbon nanotube-based devices, Sensors - Proceedings of the Second National Conference on Sensors, Rome, Italy, 19-21 February, 2014.
Banhart, F., Li, J. X., and Krasheninnikov, A. V., Carbon nanotubes under electron irradiation: stability of the tubes and their action as pipes for atom transport, Phys. Rev. B, 71, 241408-241411, 2005.

Datsyuk, V., Kalyva, M., Papagelis, K., Parthenios, J., Tasis, D., Siokou, A., Kallitsis, I., and Galiotis, C.: Chemical oxidation of multiwalled carbon nanotubes, CARBON, 46, 833-840, 2008.

Deretzis, I. and La Magna, A.: Role of contact bonding on electronic transport in metal-carbon nanotube-metal systems, Nanotechnology, 17, 5063-5072, 2006.

Donato, N., Latino, M., and Neri, G.: Novel Carbon NanotubesBased Hybrid Composites for Sensing Applications, Carbon nanotubes - From Research to Applications, 14, 229-242, 2011.

Iijima, S., Ichihashi, T., and Ando, Y.: Pentagons, heptagons and negative curvature in graphite microtubule growth, Nature, 356, 776-778, 1992.

Neophytou, N., Ahmed, S., and Klimeck, G.: Influence of vacancies on metallic nanotube transport properties, Appl. Phys. Lett. 90, 182119-182121, 2007.

Robinson, J. A., Snow, E. S., Bădescu, Ş. C., Reinecke, T. L., and Perkins, F. K.: Role of Defects in Single-Walled Carbon Nanotube, Chemical Sensors. Nano Lett., 6, 1747-1751, 2006.

Scalese, S., Scuderi, V., Bagiante, S., Gibilisco, S., Faraci, G., and Privitera, V.: Order and disorder of carbon deposit produced by arc discharge in liquid nitrogen, J. Appl. Phys. 108, 064305064309, 2010.

Scuderi, V., La Magna, A., Pistone, A., Donato, N., Neri, G., and Scalese, S.: Use of the electric fields for the manipulation of MWCNTs, Carbon-Based Low Dimensional Materials - Proceedings of the 2nd CARBOMAT Workshop ISBN 978-88-1245, 86-89, 2012a.

Scuderi, V., Bongiorno, C., Faraci, G., and Scalese, S.: Effect of the liquid environment on the formation of carbon nanotubes and graphene layers by arcing processes, Carbon, 50, 2365-2369, 2012b.

Scuderi, V., Tripodi, L., Piluso, N., Bongiorno, C., Di Franco, S., and Scalese, S.: Current-induced defect formation in multiwalled carbon nanotubes, J. Nanopart. Res., 16, 2287-2292, 2014.

Zhang, T., Mubeen, S., Myung, N. V., and Deshusses M. A.: Recent progress in carbon nanotube-based gas sensors, Nanotech. 19, 332001-332014, 2008.

Zhou, O., Shimoda, H., Gao, B., Oh, S., Flaming, L., and Yue, G.: Materials science of carbon nanotubes: Fabrication, integration, and properties of macroscopic structures of carbon nanotubes, Accounts Chem. Res., 35, 1045-1053, 2002. 